\title{
The evadable method of static state threat based on route programming in two-dimensional space

\author{
Dai Chao, He Qian
}

China Satellite Maritime Tracking and Control Department, Jiangyin, 214431, China

Keywords: Threat; avoid; two - dimensional space

\begin{abstract}
In the case where the static threat a priori position information has been obtained, the threat avoidance of the unmanned combat aircraft can be realized by the pre-route planning method. In this paper, we study the planning method of evading route under the condition of known static threat. According to the threat object of unmanned combat aircraft, the model of threat protection area is established firstly. Then, based on route planning algorithm, the unmanned combat aircraft can avoid the static threat in two-dimensional space.
\end{abstract}

\section{Introduction}

Unmanned combat aircraft in the implementation of various types of combat missions, inevitably encounter such as early warning radar, ground-to-air missile positions, artillery positions and other threats. This paper assumes that the location information of the threat source in the battlefield environment has been detected by the reconnaissance UAV and calibrated on the map.

\section{Threat sources}

When there is a single threat source between the UAV and the target point, the UAV is limited to the left or right side of the threat in the 2D plane, as shown in Figure 1. The task of unmanned combat airplane threat avoidance is to select a route which satisfies the kinematic characteristics of unmanned combat aircraft and deviates from the smallest route of the original route from all routes that can successfully avoid the threat and makes the unmanned combat aircraft fly to the target point along the route.

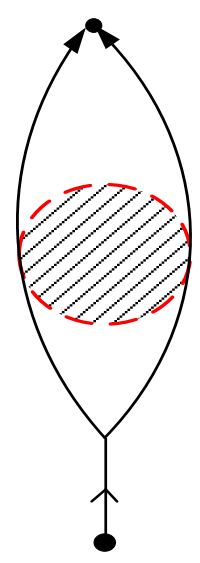

Figure 1. Threat Avoidance in 2D Plane

Unmanned combat aircraft need to avoid the main threat object shown in Figure 2. 


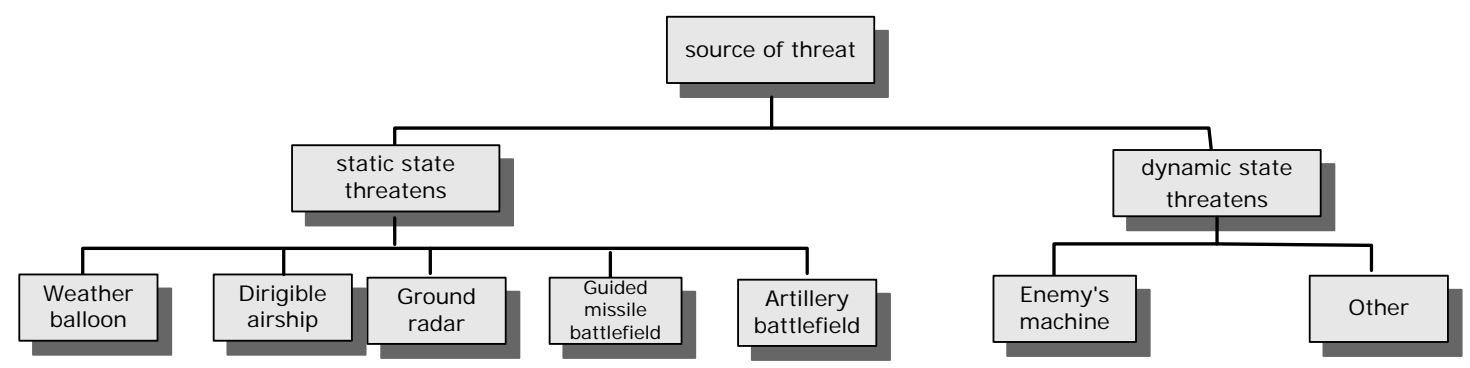

Figure 2. Threat object classification

According to the above classification of threat objects, we should first establish the corresponding threat model, and then take appropriate methods to avoid conflicts with such objects.

The Threat Protection Area describes the no-fly zone in the unmanned combat aircraft during flight. The design of the protected area should reflect the minimum safe distance of the unmanned combat aircraft from the threat, taking into account the spacing requirements of the threat aversion space. If the protected area is invaded, it is considered that the unmanned combat aircraft's strategy to avoid the threat has failed. Two key characteristics of the threat protection area are size and shape. When focusing only on horizontal avoidance maneuvers and velocity changes, in two-dimensional space, the protected area is usually represented by a circle centered on the threat center, as shown in Fig.

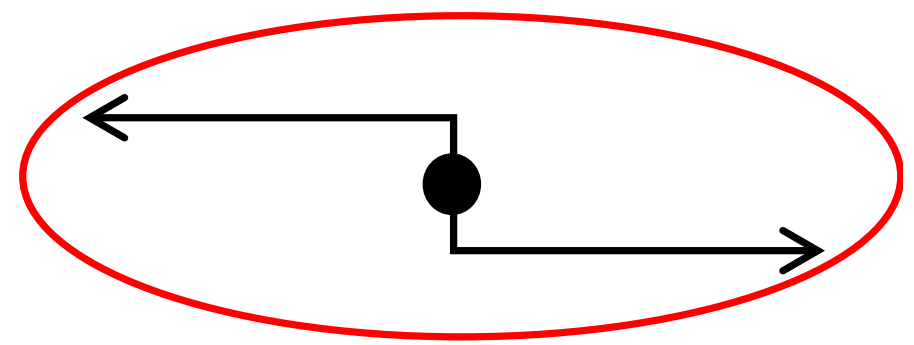

Figure 3. Threat in the two - dimensional space

\section{Route planning formula}

One of the constraints of route planning is the kinematics of the UCAV itself. Kinematic characteristics The unmanned combat aircraft's velocity vector is obtained by combining the lateral and roll acceleration vectors of the unmanned combat aircraft. Using the attitude angle of the unmanned combat aircraft and its current position in the inertial frame, The speed of the plane and the roll of the combat aircraft. Think of unmanned combat aircraft as a control system. The unmanned combat aircraft system has two circuits, the inner loop is the autopilot loop and the outer loop is the guidance loop. The guidance module provides lateral acceleration commands to enable the unmanned combat aircraft to track the intended course, and the autopilot controls the unmanned aircraft's rudder surface to achieve the required lateral acceleration.

The unmanned combat aircraft kinematics model is used for the route planning in the two-dimensional plane:

$$
\left\{\begin{array}{l}
\dot{x}=V \cos \theta \\
\dot{y}=V \sin \theta
\end{array}\right.
$$

In the formula, $\mathrm{v}$ is the speed of unmanned combat aircraft, and $\theta$ is the horizontal heading angle of unmanned combat aircraft.

Planning to generate the route to meet the UAV's kinematic radius of curvature constraints. To meet this constraint, the unmanned combat aircraft movement must be limited to maximum lateral acceleration. The relationship between the radius of curvature and the lateral acceleration is described by equation (2). The radius of curvature at any point on the route must be greater than the minimum turning radius of the unmanned combat aircraft.

$$
a_{c}=\frac{V^{2}}{\rho}
$$

The second constraint on route planning is the threat in the battlefield environment. Since an 
unmanned combat aircraft is an in-flight route generated in accordance with route planning, the route must avoid threats in the environment. Equation (2) may be modified to be of the form:

$$
P_{s i}\left(x_{s i}, y_{s i}, z_{s i}, \theta_{s i}, \psi_{s i}\right) \stackrel{\amalg_{s f i e}, \amalg_{p}, r_{i}(q)}{\longrightarrow} P_{f i}\left(x_{f i}, y_{f i}, z_{f i}, \theta_{f i}, \psi_{f i}\right) \quad i=1,2, \cdots N
$$

\section{Local route planning for two - dimensional static static threat avoidance}

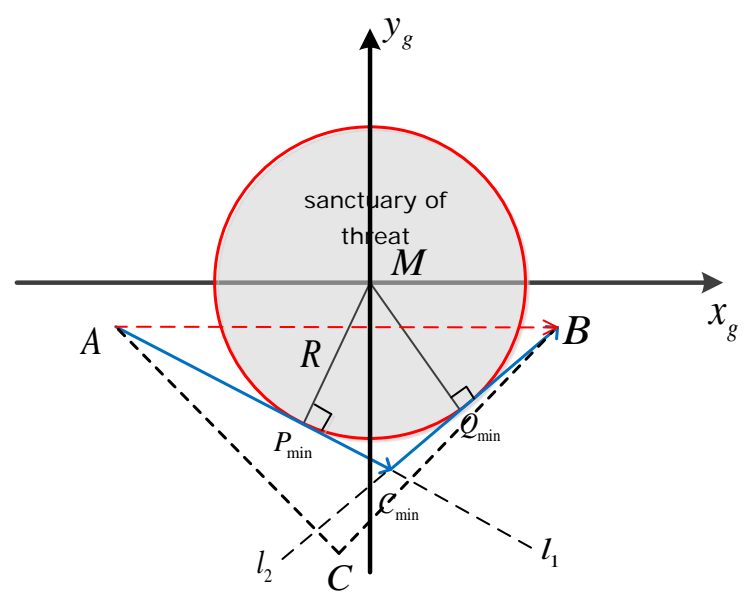

Figure 4. Two - dimensional space for a single static threat to avoid the local route planning diagram

It is easy to see that if an unmanned combat aircraft flew to the target point B along the straight line from the starting point $\mathrm{A}$, it must pass through the protected area of threat $\mathrm{M}$, that is, the collision should be planned, so that a flight suitable for unmanned combat aircraft A certain condition of the route, so that unmanned combat aircraft successfully avoid the threat to reach the target point.

Get the coordinates of the middle way point, which is completed on the unmanned combat aircraft from the starting point $\mathrm{A}$ to the target point $\mathrm{B}$ route planning.

\section{Simulation verification and result analysis}

An unmanned combat aircraft based on the pre-route planning method is used to simulate a single known threat. The main parameters of the unmanned combat aircraft used in the simulation calculation are shown in Table 1, and the six-degree-of-freedom model is used to characterize the motion characteristics of unmanned combat aircraft.

Table 1. Unmanned combat aircraft parameters

\begin{tabular}{cccc}
\hline quality & $17 \mathrm{Kg}$ & Wing area & $1.3536 \mathrm{~m}^{2}$ \\
Wingspan & $3.2 \mathrm{~m}$ & Average chord length & $0.423 \mathrm{~m}$ \\
Cruising speed & $25 \mathrm{~m} / \mathrm{s}$ & Match the angle of attack & $2.35 \mathrm{deg}$ \\
\hline
\end{tabular}

The guidance period is $0.5 \mathrm{~s}$, and the minimum turning radius of the unmanned combat aircraft is $r_{\min }=175.22 m$ by the coordinated turning formula: ${ }_{\min }^{r_{\min }=\frac{V_{c}^{2}}{g \tan \left[\left(\phi_{c}\right)_{\max }\right]}}$. The center of the threat is the origin of the ground coordinate system. The radius of the threat zone is $R=550 \mathrm{~m}\left(\mathrm{R}>\mathrm{r}_{\min }\right)$. The coordinates of the starting point $A$ of the unmanned combat aircraft are $(-1500,-100)$ and the position of the target point $B$ is $(1000,-100)$. (50.974, -593.39$)$, the coordinates of the route middle point $C$ calculated from the previous analysis are (50.974, -593.39).

Through simulation, the unmanned combat aircraft flight path is shown in Figure 5. 


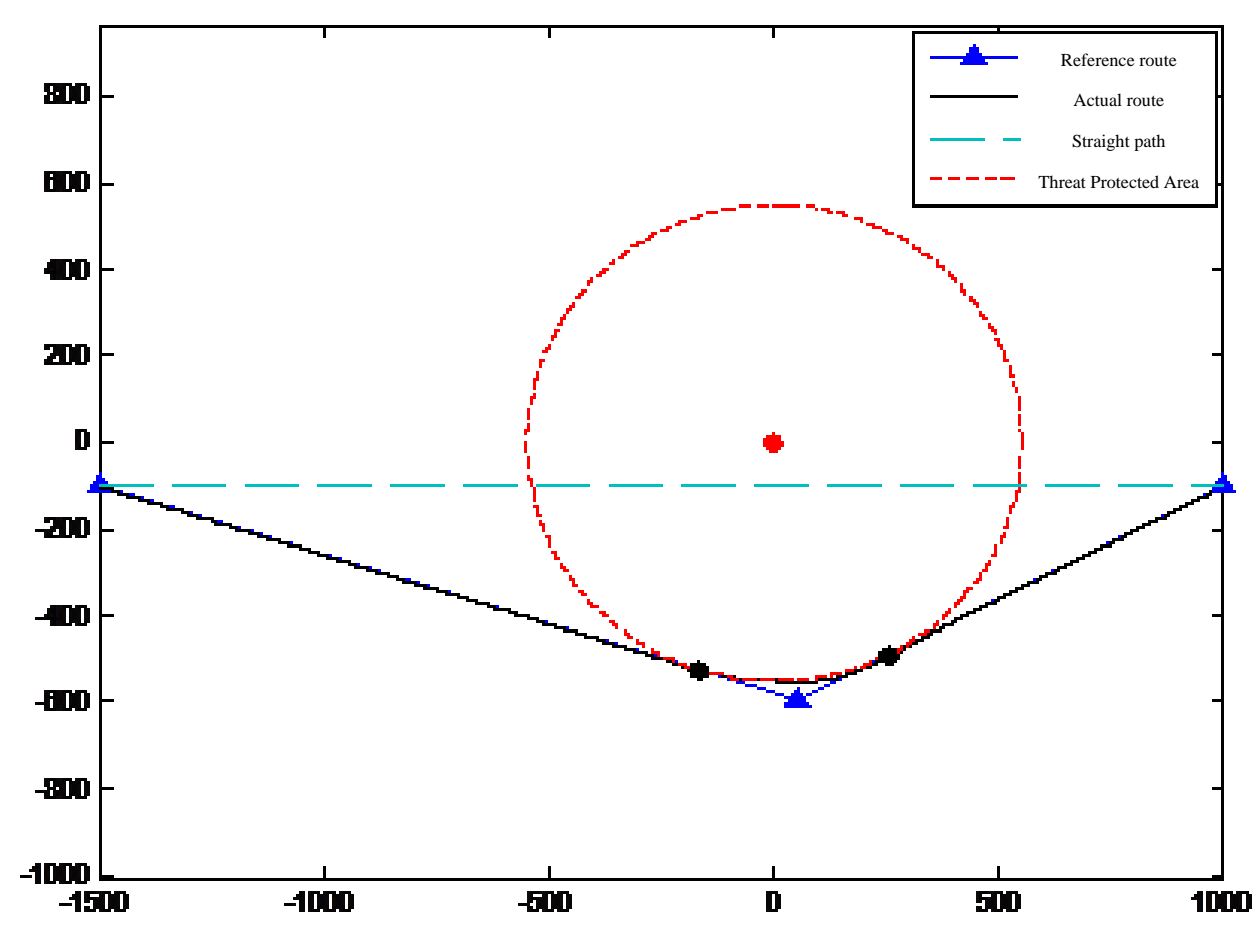

Figure 5. The actual flight path of unmanned combat aircraft

The red dotted line in Fig. 5 is the threat area, and the unmanned combat aircraft is safe to fly outside the threatening area. The blue dotted line is the straight line between the starting point and the target point. It can be seen that, before reaching the point of entry, the unmanned combat aircraft track is almost coincident with the planned route, after which it follows the safety boundary turn and reaches the cut-off point and continues along the pre-planned route aims.

\section{Summary}

In this paper, the basic theory and formula of route planning are introduced after the establishment of the threat protection model. The shortest voyage cost is the key to analyze the two-dimensional and three-dimensional space based on route planning to avoid known static threats. The middle way point that the aircraft avoids the threat and simulates the evasion of the static threat in the two-dimensional space.

\section{Reference}

[1] Wei Ruixuan, Li Xueren. UAV system and operational use [M]. Beijing: National Defense Industry Press, 2014: 1-25.

[2] Shen Lincheng, Niu Yifeng. Multi-UAV Autonomous Cooperative Control Theory and Method [M]. Beijing: National Defense Industry Press, 2013: 1-107.

[3] Mao Hongbao, Tian Song. UAV mission planning [M]. Beijing: National Defense Industry Press, 2015: 76-82. 